\title{
A simulation-assisted complexity metric for design optimization of integrated architecture aero-engine structures
}

\author{
Visakha Raja ${ }^{1,3}$ - Michael Kokkolaras ${ }^{2,3} \cdot$ Ola Isaksson $^{3}$ \\ Received: 21 December 2018 / Revised: 1 May 2019 / Accepted: 6 May 2019 / Published online: 23 May 2019 \\ (C) The Author(s) 2019
}

\begin{abstract}
Traditional metrics for assessing system complexity are based on aspects such as number of and interaction among components. For functionally integrated structures, the application of such metrics can be difficult and/or limited due to the inseparability of the structure into components or sub-systems; a single monolithic structure satisfies all required functions. At the same time, complexity metrics are necessary for effective application of design optimization and systems engineering principles. Aero-engine static structures are typical examples of functionally integrated architecture. In this paper, we present a complexity metric for integrated architecture structures that can be included as an objective or constraint in design optimization problem formulations. The proposed metric is based on two existing metrics, one providing a system wide scheme for complexity calculations and the second, giving complexity for individual components. In order to account for its integrated architecture, different regions of the structure are considered. Interactions are estimated as load paths through the structure, identified by means of physical simulations. Complexity evaluations are demonstrated using two detail-designed aero-engine structures with similar functions but belonging to different engine designs. Despite the similarities, the structures differ in complexity. This enables quantitative comparison among different designs of integrated architecture structures based on physical arrangements and main functions. Moreover, the metric can be used to identify regions with most influence on complexity which in turn enables design improvements on those regions. Automated computation of the metric can result in rapid comparison and selection among a number of structure designs, and thus be used in optimization studies. Finally, a correlation of the metric with the development time or cost can be useful for future integrated architecture structure design optimization.
\end{abstract}

Keywords Engineering design $\cdot$ Structural complexity $\cdot$ Topology optimization $\cdot$ Aero-engine structures

Responsible Editor: Erdem Acar

Visakha Raja

visakha@chalmers.se

Michael Kokkolaras

michael.kokkolaras@mcgill.ca

Ola Isaksson

ola.isaksson@chalmers.se

1 GKN Aerospace Sweden AB, Trollhättan, Sweden

2 Department of Mechanical Engineering, McGill University, Montreal, Canada

3 Department of Industrial and Materials Science, Chalmers University of Technology, Göteborg, Sweden

\section{Introduction}

The term product architecture is used to describe how physical components accomplish desired functions. In an aero-engine, most structural components are functionally integrated meaning that all required structure functions are satisfied by a single, monolithic component. Such products are called integrated (as opposed to modular) architecture products (Raja 2016).

The design philosophies for the products can be varied. For example, in decision-based engineering design (Hazelrigg 1998), several alternative architectures for the products are generated and compared with each other to select the most suitable alternative. In set-based concurrent engineering (Sobek et al. 1999), a set of architectures are 
considered and each member in the set is systematically eliminated to converge to a limited number of choices. Irrespective of design philosophies, quantitative metrics are necessary for the comparison, selection, or elimination of alternatives. The metrics can enable rapid evaluation among several designs. Various measures of product complexity can be used to achieve the selection.

Reducing complexity has been reported (Pugh 1990) to result in better product designs. In the engineering design literature, several measures of complexity are available (ElMaraghy et al. 2012). However, the direct application of such metrics is often not possible since they are developed for multi-part products or multi-components systems. For integrated products, such as aero-engine structures, the terms, part, component, or system take the same meaning and no physical separation is possible. There is a need for complexity metrics applicable to such integrated engine structures in the aerospace industry, so that different entry into service (EIS) design configurations can be compared.

Typical examples of integrated architecture products are static structures located between two engine modules such as the low pressure compressor (LPC) and the high pressure compressor (HPC). While the structures are seemingly similar, design features vary considerably both in appearance and number within the same product class. Figure 1 depicts two such structures (part of the compressor module in two different aero-engine designs).

The main function of the structures consists of transferring engine core flow between modules and withstanding mechanical loads originating from its interfaces with the modules. Thus, the two main aspects of the architecture of the structures are their gas flow path and mechanical load path. Any static structure, irrespective of the engine to which it belongs, shares the gas flow path and mechanical load path characteristics. An appropriate complexity metric should include aspects of fundamental functions of the structures in addition to the physical arrangement of the product.

The purpose of this paper is to derive a complexity metric for functionally integrated aero-engine structures. As the integrated structure cannot be split into components, different regions are considered instead of components and interactions are estimated as load paths through the structure from physical simulations for different loading scenarios in the structure.

\section{Literature review}

The concept of complexity is used in several design engineering contexts in different ways (Chu et al. 2003; Maurer et al. 2014). Complexity is often described in terms of various metrics based on the number and interactions of distinct components or functions of a system (Shah and Runger 2013). For a product, complexity can arise from internal (increased product variety) or external sources (Lindemann et al. 2009). According to Weber (2005), in product development and engineering design, there exist five dimensions of complexity. These are numerical (related to the number of components), relational/structural (relations and interdependencies among components), variational (number of variants of the product), disciplinary (number of disciplines involved in creating the product), and organizational (related to how the development organization is structured). The first three dimensions (numerical, relational, variational) may be readily associated to the physical characteristics of a product. In contrast, Suh (2005) argues for defining complexity only in the functional domain. Complexity is seen as the uncertainty in achieving the functional requirements of a product. Therefore, a product's complexity metric can be based on either its physical or functional characteristics.

The architecture of a product affects how complexity is viewed and managed. Many firms adopt modularization to
Fig. 1 Two static compressor structures for two different engines. Even though the functions and the external appearance are comparable, individual design features are different
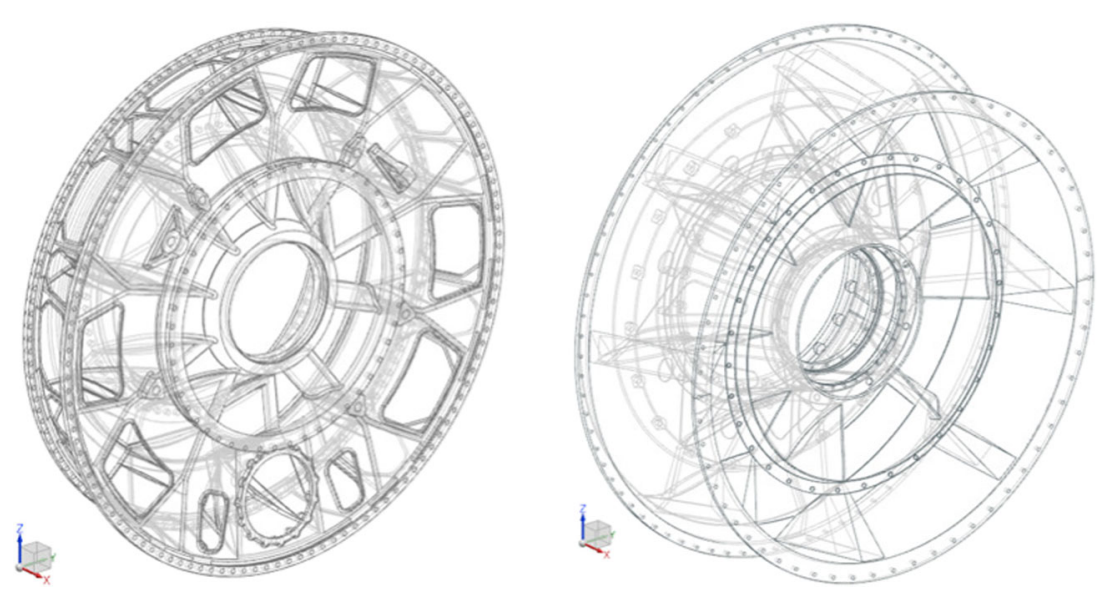
manage complexity (Jiao et al. 2007). Complexity can be also expressed directly, without explicitly considering modularity. For example, Summers and Shah (2010) classify complexity metrics in engineering design into three categories. Metrics developed for design problems (statement of objectives and requirements), design processes (steps taken to achieve the design problem objectives), and design products (the final result of carrying out the design processes). This can be viewed as an assessment of complexity during the different stages of product development.

The physical form of the engine structures considered here are detail-designed and final. The structures belong to the last stage of development. From Weber's dimensions for complexity, physical products can have numerical, relational, and variational dimensions. A metric for design products that satisfies these dimensions will be appropriate for the structures. Additionally, metrics of modularity are generated based on physical products and can be considered as complexity metrics belonging to the design products category.

When complexity is managed through product modularity, a number of metrics are available to assess the it. Mikkola (2006) proposes a modularization function dependent on the type of components, interfaces, degree of coupling and substitutability. Values of the modularization function can vary between 0 and 1 and the higher the function value, the higher the modularity (or the lesser the complexity). Hölttä-Otto and de Weck (2007) introduce singular value modularity index (SMI) for assessing degree of modularity. The index is based on singular value decay patterns for the respective product DSMs. Similar to the modularization function, SMI also varies between 0 and 1 and a larger value of SMI indicates higher modularity. For both metrics, not considering the type of interaction among product components is a drawback.

For design products, two types of complexity are proposed by Ameri et al. (2008): size complexity (a measure of information content within product representation) and coupling complexity (a measure based on the coupling between the types of nodes in a bi-partite graph). Size and coupling complexities are evaluated based on three representations. A parametric associativity graph (PAG) that associates different dimensional parameters to a product's part, the function structure that indicates the interrelation of different functions of a product and connectivity graphs that represent the connections and the type of connections such as press fit or snap fit among components. The metric proposed by Sinha and de Weck (2016) considers the type of interaction among individual system components. Sinha and de Weck's metric is composed of three separate constituents: (i) a components complexity $C 1$, attributed to the complexity of individual components; (ii) an interface complexity $C 2$, attributed to the different types of interaction among system components; and (iii) topological complexity $C 3$, attributed to the specific layout of the system under consideration. The metric is validated against theoretical criteria and has had applications in the evaluation of product platform complexity (Kim et al. 2016) and the level of decomposition in a system (Min et al. 2015).

From the foregoing discussions, it can be observed that all metrics are developed for multi-part products or multicomponent systems. Application of such metrics has not yet been demonstrated on integrated architecture products. Thus, it is necessary to develop a complexity metric that is directly applicable to integrated architecture products.

\section{Complexity of integrated architecture structures}

The complexity metric in this paper is derived through modifying existing metrics that are applicable only to multipart products or multi-component systems. The structures are assumed to consist of a number of sections (Raja and Isaksson 2015), which are identifiable regions of the structure accomplishing possibly different functions. One or more sections may be associated with accomplishing a function. For example, consider a coffee cup that has an ear and a cylinder. These two sections of the cup structure accomplish the functions of letting the cup be held and holding the coffee respectively. Similar to structures, features such as bolt holes and seal surfaces have been used to associate physical regions with an identifiable shape in reverse engineering (Urbanic and ElMaraghy 2009). Sections in a structure are geometrically more elaborate than bolt holes or seal surfaces, requiring several dimensional parameters to fully describe them.

When an integrated architecture product is partitioned into sections, the numerical (number of sections), variational (variation among different sections), and relational (sectional interaction through load transfer) dimensions of Weber's complexity criteria are applicable. The metric by Sinha and de Weck (2016) allows for both the architecture as well as interactions in the system to be included in the same metric. Therefore, we use it as the foundation of our metric. At the same time, one issue we have to address is the calculation of complexities of the different sections. If the sections of the structure are simplified, complexity can be calculated following the method proposed by Ameri et al. (2008). Thus, the complexity metric developed in this paper for integrated architecture structures is based on modifying and integrating these two metrics.

Section 3.1 describes the generic scheme of complexity calculations for integrated architecture structures, and Section 3.2 demonstrates the calculations on two model structures. 


\subsection{Complexity calculations}

According to Sinha and de Weck (2016), complexity can be calculated by the following:

$C=C 1+C 2 C 3$

where $C$ is the total complexity of the system, $C 1$ is the complexity of the individual components (called components complexity), $C 2$ is the complexity due to inter component interactions (called interface complexity), and $C 3$ is the complexity due to the component layout or architecture (called topological complexity) of the system.

The first constituent of complexity, $C 1$, is the sum of complexities of the system components. Here, system is the engine structure and system components are the different sections of the structure. $C 1$ for a section is computed from the size complexity (measure of information content in a representation; expression follows) for its PAG. A PAG contains two types of nodes. The parts of a product and the geometrical dimensions for the parts. The edges of the PAG can also be of two types, part-dimension and dimensiondimension. For example, a cylinder as shown in Fig. 2a has dimensions for its length and radius. The corresponding PAG in Fig. 2b has two types of nodes represented by rectangles and ovals. These are part type (the cylinder) and dimensions. The part type requires two dimensions to fully define it. The size complexity (Ameri et al. 2008) based on the PAG is calculated using (2).

$C_{\mathrm{PAG}}=\left(n_{\text {nodes }}+n_{\text {edges }}\right) \ln \left(n_{\text {node types }}+n_{\text {relation types }}\right)$,

where $n_{\text {nodes }}$ are the total number of nodes in the PAG, $n_{\text {edges }}$ is the total number of edges, $n_{\text {node types }}$ is the total number of types of nodes, and $n_{\text {relation types }}$ is the total number of types of relations in the PAG. The complexity for the cylinder in Fig. 2a is as follows:

$C_{\mathrm{PAG}}=(3+2) \ln (2+1)=2.39$

While calculating $C 1$ complexity for the sections, the dimension-dimension aspect is not considered and each section is treated as being independent.

The second constituent of complexity, $C 2$, is the complexity due to the interaction of components in a

(a)

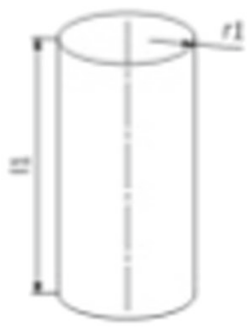

Fig. 2 a CAD model of the cylinder with dimensions; b PAG for the cylinder with dimensions system. The interactions can be the flow of material, energy, or signals/information (Pahl et al. 2007). Accordingly, $C 2$ is calculated as follows:

$C 2=\sum_{i=1}^{n} \sum_{i=1}^{n} \beta_{i j} A_{i j}$

where $A_{i j}$ are the elements of the respective adjacency matrix for each type of interaction, $\beta_{i j}$ are the complexities associated with each interaction, and $n$ is the number of components in the system. The elements $A_{i j}$ of the $n \times n$ adjacency matrix is defined as follows:

$A_{i j}=\left\{\begin{array}{l}1 \text { if }(i \neq j) \text { and components } i \text { and } j \text { interact } \\ 0 \text { otherwise }\end{array}\right.$

For an integrated structure, no material, energy or signal flows through the bulk of the structure. The mechanical loads that the structure carries can be considered as a variant of energy flow. Identification of load paths in the structure corresponding to different loading scenarios establishes sectional connectivity similar to energy flow through different components in a system. This enables the calculation of $C 2$ complexity. The complexity of each pairwise interaction between components, $\beta_{i j}$, is assumed to be 0.5 with each interacting section having equal importance in transferring the load. A detailed description of $C 2$ calculation on an illustrative example is given in Section 3.2.

The third and last constituent of complexity, called topological complexity (C3), can be used to represent the architecture of the system. It is a global measure, calculated based on the aggregated adjacency matrix for the component interactions. Here, $C 3$ is based on a mechanical connectivity matrix. Elements of the matrix are defined similarly to (4); unity if section $i$ of a structure is connected to section $j$ and 0 otherwise. The rows and columns of the matrix originate from a union of the different sections present in all the considered structures. Say that there are $m$ structures and $S_{s 1}, S_{s 2}, \ldots, S_{s m}$ are the sets of sections for the structures. The set of sections $S$ in the columns (or rows) of the mechanical connectivity matrix can be written as follows:

$S=\bigcup_{i=1}^{m} S_{s i}$

If $k$ is the number of elements in the union set $S$, the expression for $C 3$ can be written as follows:

$C 3=\frac{1}{k} \sum_{i=1}^{k} \sigma_{i}$

where $\sigma_{i}$ is the $i$ th singular value of the sectional adjacency matrix. Detailed description of $C 3$ calculation is given in Section 3.2. 


\subsection{Illustrative example}

To illustrate the complexity calculations, two load carrying structures, "design-01" and "design-02" are considered as shown in Fig. 3. It is assumed that all required functions are satisfied by one single monolithic structure and the two alternative designs satisfy similar type of functions in similar environments. Thus, the two designs can be said to belong to the same "class" of products.

\subsubsection{Components complexity or C1 complexity}

To calculate $C 1$, the structures need to be divided into sections. The sections present in design-01 and design02 are marked in Fig. 3. The sectional division for the model structures is ad-hoc, following existing conventions at the design firm. If the same structures are designed at another firm, a different geometry might describe a section and the resulting complexities will differ. However, within one firm, the sectional division conventions for a certain class of products are assumed to be uniform. Once the sections have been identified, individual sectional complexities of the structures can be calculated. Figure 4 shows the dimensioning for unique individual sections, the associated complexities for the sections and the total values of $C 1$ complexity for design- 01 and design- 02 .

\subsubsection{Interface complexity or $C 2$ complexity}

The interface complexity, $C 2$, depends on component interactions in a system. For an integrated architecture product, the interactions are among the different sections of the product in the form of load path passages. The load path in a structure is a visualization of the load transfer from the point of application of the load to the point of support. A number of approaches exist to identify load paths in a structure. Kelly et al. (2011) proposes a method to plot contours for which load in a certain coordinate direction remains constant, from the load application point to the support point. Another approach is to plot contours of the strength of connection (called a stiffness index) between the loaded points and non-loaded points in the structure, which are representative of the load paths (Shinobu et al. 1995; Naito et al. 2011). Commercial implementation of both the methods are not easily available which is necessary for industrial usage. Here, an approach based on topology optimization using a commercial software (OPTISTRUCT 2017) is used for finding the load path.

A commonly used objective in structural optimization is the minimization of compliance. Compliance is a reciprocal measure of stiffness. The compliance minimization optimization seeks to maximize a structure's stiffness by varying individual elemental densities from zero to the actual material value, satisfying a certain lower mass limit for the design volume. The result is a series of hollow and material filled regions within the design volume. If the objective is changed to minimizing the mass of the structure, so that it has a certain maximum compliance, all non stiffness-essential material presence will be eliminated from the structure. The regions of material left in the structure will be indicative of the load path. This principle is utilized in the load path identification.

In this work, a two-step process is used for identifying the load path of integrated architecture structures. In step 1 , the compliance of the structure under a given loading scenario is determined. In step 2, a mass minimization structural optimization is performed under the same loading scenario. A 5\% higher value of compliance from step

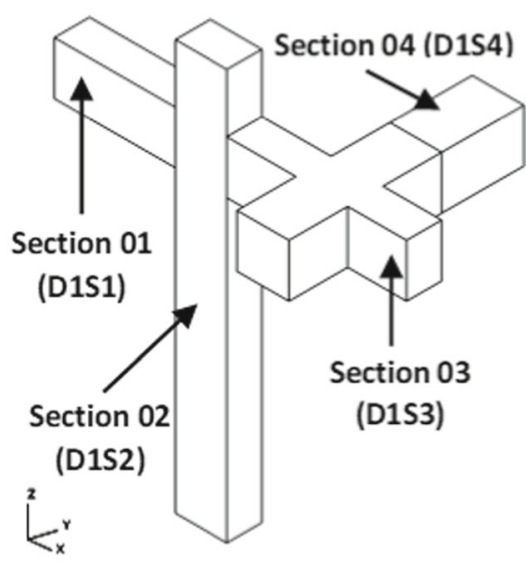

a) b)

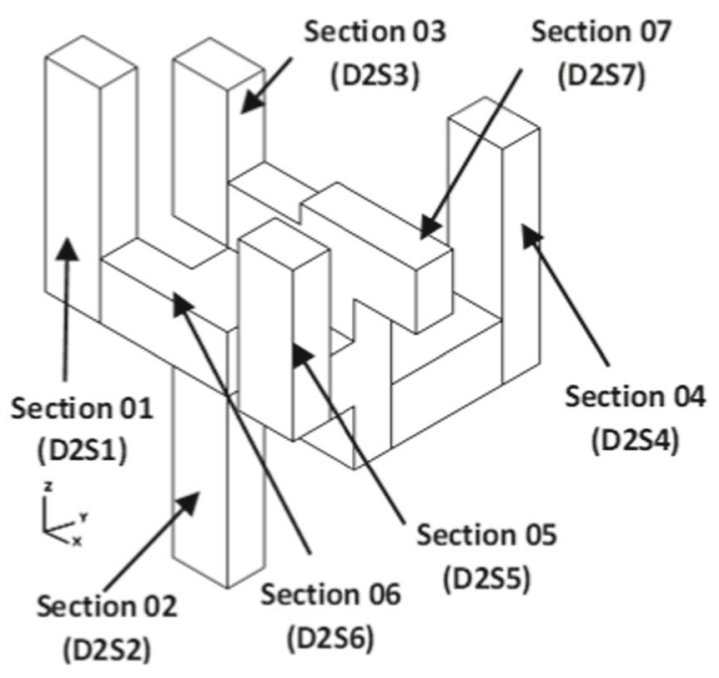

Fig. 3 Two different load carrying structure designs a design-01 and b design-02 

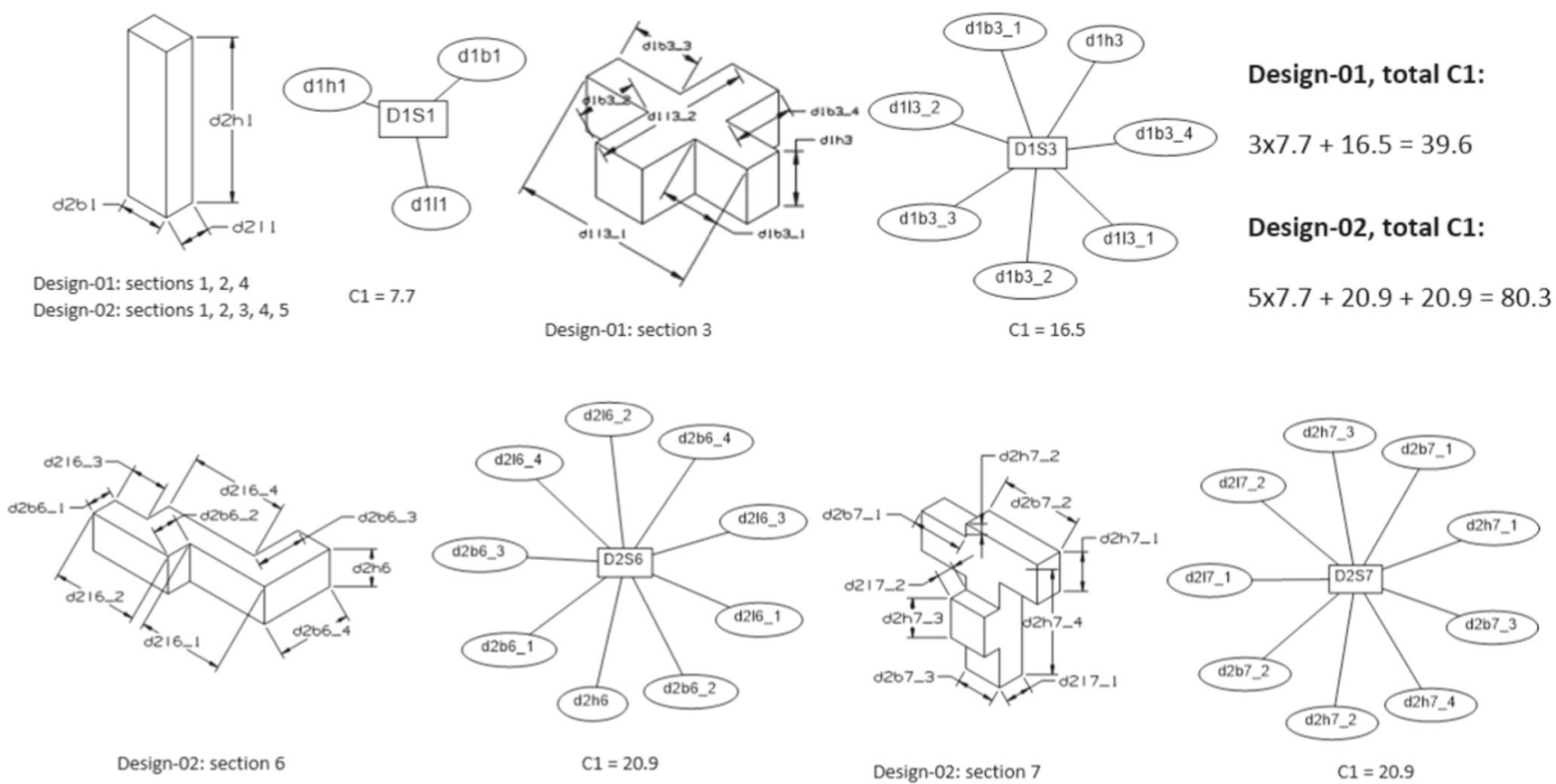

Fig. 4 Individual sectional complexities and total $C 1$ complexity for design-01 and design-02. Only geometrically unique sections are shown

1 provides an upper limit constraint in the optimization. The 5\% higher value is specified because this in effect causes a marginal relaxation in the required stiffness of the structure which helps to initiate material removal from non stiffness-essential regions. The design variables are elemental densities for all elements except those at the loading and support regions. The optimization problem can be expressed as follows:

$$
\min _{\rho} M(\rho)
$$

subject to

$$
\begin{aligned}
L(\rho) & \leq 1.05 L_{1} \\
\mathbf{0} \leq \boldsymbol{\rho} & \leq \boldsymbol{\rho}_{\text {material }}
\end{aligned}
$$

where $\boldsymbol{\rho}=\left[\rho_{1}, \rho_{2}, \ldots, \rho_{k}\right]$, is the vector of densities for each element and $k$ is the total number of elements included in the optimization. $M(\rho)$ and $L(\rho)$ are the mass and compliance of the structure, based on the elements included, and $\rho_{\text {material }}$ is the structure's material density. $L_{1}$ is the compliance of the structure obtained from step 1 .

After the optimization, the locations in the structure where the elemental density is more than $75 \%$ of the actual value for the material are noted. The locations will be representative of the load path in the structure, for the considered loading scenario. The interface complexity $C 2$ can then be determined by identifying the sections at which material is left after optimization. There will be as many $C 2$ metrics as many load cases and the total $C 2$ for the structure will be the sum of all load case specific $C 2$ metrics. For the structures considered here, two loading scenarios were considered. For both scenarios, the same analysis boundary conditions were used which is to fix the component in all
Fig. 5 Loading scenarios on the different designs a design-01 and $\mathbf{b}$ design-02. LS1 load has blue arrows and LS2 load has red arrows. Both loading scenarios have the same boundary conditions

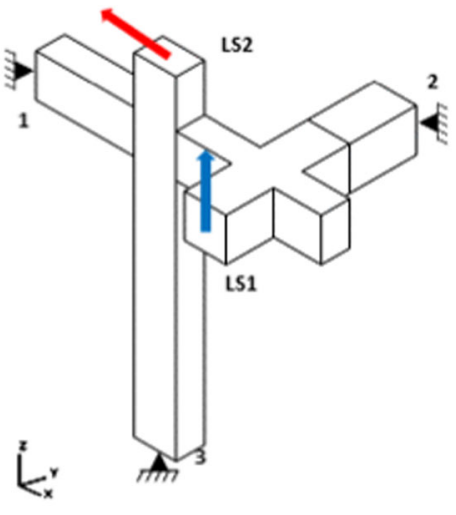

a)

b)

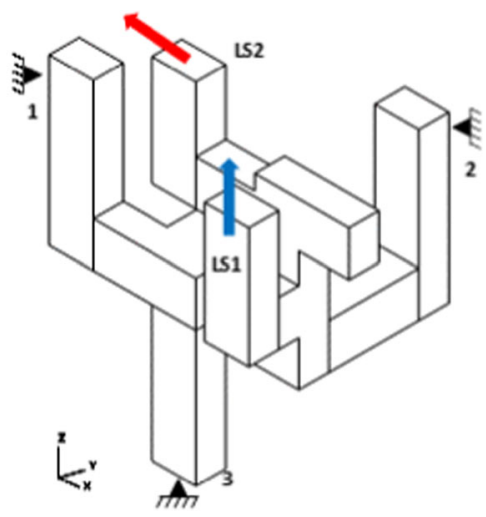


a)

\section{Contour Plot}

Element Densities(Density)

Advanced Average

1.00
-0.75
0.01

Isq.8.75

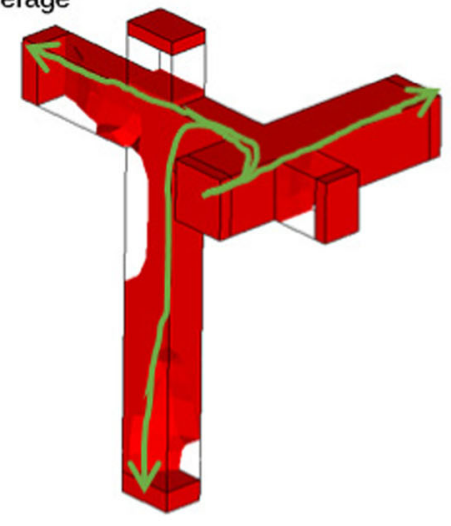

Contour Plot

Element Densities(Density)

Advanced Average

c)

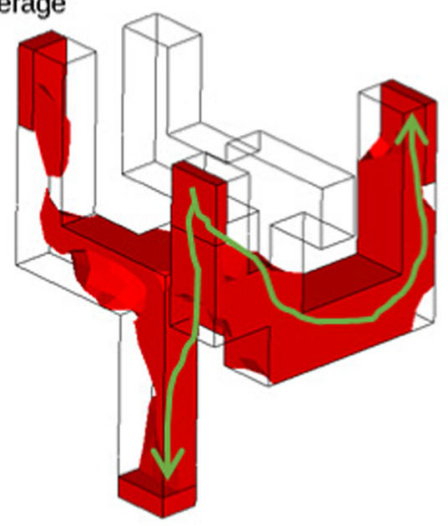

b)

\section{Contour Plot}

Element Densities(Density)

Advanced Average

T 1.00
0.75
0.01
Isco.75

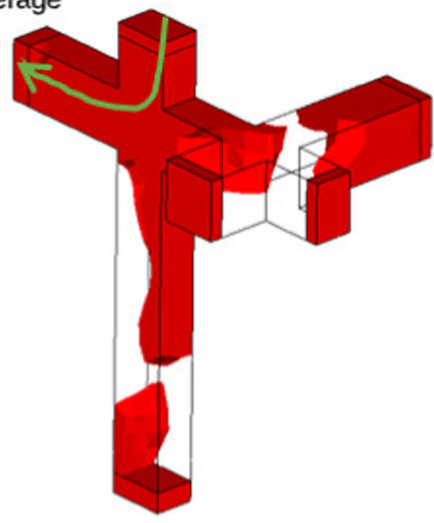

Contour Plot

Element Densities(Density)

Advanced Average

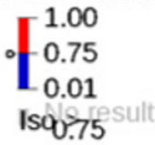

d)

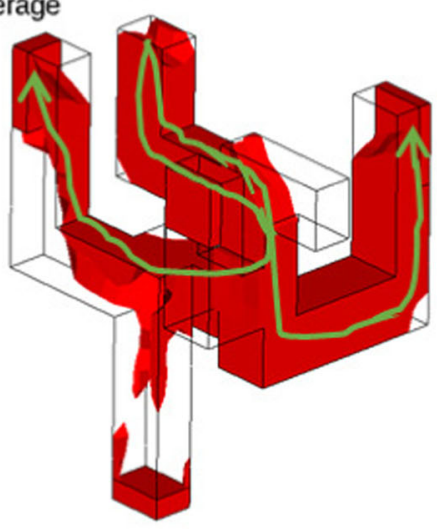

Fig. 6 Resulting load paths for the example structures. The load paths are indicated using directed free drawn green lines. a LS-1 for design-01 and 02 b LS- 2 for design-01 c LS-1 for design-02 d LS-2 for design-02

directions at points 1, 2, and 3 (cf. Fig. 5). The first scenario is the application of a vertical load (blue arrow, indicated as LS1 in Fig. 5a, b) and the second, a horizontal load (red arrow, indicated as LS2 in Fig. 5a, b).

Load paths for the two loading scenarios were identified using the procedure described above and the result of the structural optimization where at least $75 \%$ of the material (that is, elemental density is at least $75 \%$ or more) is retained is shown in Fig. 6. In the figure, continuous material trails represent load paths. For example, three continuous trail of material can be discerned from the loading point to the support points during LS1 for design-01 (Fig. 6a) and all sections in participate in the load transfer. During LS2 for the same design (Fig. 6b), continuous material trail exists only to the support point 1 and barely two sections participate in the transfer of load. Thus, the involvement of
Design-01 LS1

\begin{tabular}{|c|c|c|c|c|}
\hline & D1S1 & D1S2 & D1S3 & D1S4 \\
\hline D151 & 0 & 0 & 0 & 0 \\
\hline D1S2 & 1 & 0 & 0 & 0 \\
\hline D1S3 & 0 & 1 & 0 & 1 \\
\hline D154 & 0 & 0 & 0 & 0 \\
\hline
\end{tabular}

C2_LS1 $=$

$3 \times(0.5 \times 1 \times 1)=1.5$
Design-02 LS1

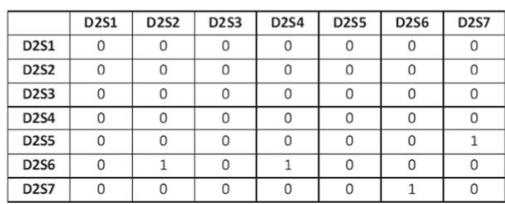

C2_LS1 =

$4 \times(0.5 \times 1 \times 1)=2.0$
Design-01 LS2

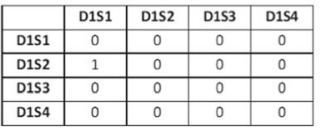

C2 2 LS2 $=$ $1 \times(0.5 \times 1 \times 1)=0.5$
Design-02 LS2

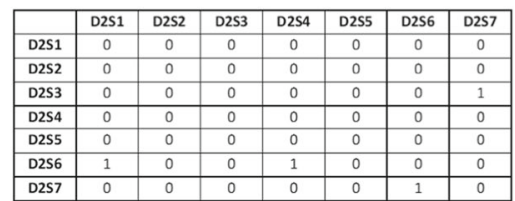

C2_LS2 =

$4 \times(0.5 \times 1 \times 1)=2.0$

Fig. 7 C2 complexities for design-01 and design-02 during LS1 and LS2 
Table 1 Total $C 2$ complexity for design- 01 and design- 02

\begin{tabular}{llll}
\hline Design & $C 2$ in LS1 & $C 2$ in LS2 & Total $C 2$ \\
\hline Design-01 & 1.5 & 0.5 & 2.0 \\
Design-02 & 2.0 & 2.0 & 4.0 \\
\hline
\end{tabular}

sections in load transfer depends on the loading scenario and the number of sections involved will affect the $C 2$ complexity. All identifiable load paths for both designs during the two loading scenarios are marked with green lines in Fig. 6. The respective adjacency matrices resulting from the load path analysis are given in Fig. 7. Based on the matrices shown in Fig. 7, the computed values for $C 2$ complexities are given in Table 1.

Design-01 has smaller $C 2$ complexity as lesser number of sections participate in the load transfer compared to design-02. Additionally, the sections in design-01 are simpler which results in a shorter load path that in turn reduces the complexity. Reduction in complexity due to shorter load paths is also reported by Pugh (1990) although the scheme of complexity calculation is different in Pugh's case.

\subsubsection{Topological complexity or C3 complexity}

Topological complexity is a global measure based on the aggregated adjacency matrix for component interactions. In our case, it is based on the mechanical connectivity of sections in the structure. Since the two designs belong to the same product class, the connectivity matrix is formed such that the rows and columns of the matrix contain all sections from design-01 and design-02. For example, consider the connectivity matrix for design-01 in Fig. 8. The rows and columns of the matrix include all sections for design- 01 and design-02 (Fig. 3 depicts the sections). The resulting $C 3$ for design-01 and design-02 are given in Table 2 .

The topological complexity for design- 02 is higher than that for design-01. This is because the number of sections and the number of sectional interconnections in design-02 is more than that in design-01.
Table $2 C 3$ complexities for design-01 and design-02

\begin{tabular}{ll}
\hline Design & $C 3$ \\
\hline Design-01 & 0.56 \\
Design-02 & 0.83 \\
\hline
\end{tabular}

\subsubsection{Total complexity for the illustrative example}

After calculating $C 1, C 2$, and $C 3$, the total complexity $C$ for design-01 and design-02 can be calculated according to (1). Table 3 shows the calculations.

The total complexity for design-02 is approximately twice as that for design-01. This fact might have been apparent from their physical appearance but specifying a quantitative metric of complexity enables comparing them. For example, for design-01, most of the interface complexity $C 2$ arises due to LS1 while for design-02, $C 2$ is the same for both LS1 and LS2. If the share of a certain loading scenario is more, it uses a larger number of sections and reducing the complexity of the sections can reduce the overall complexity, resulting in a more functionally efficient structure. In general, design improvements for design-01 may be directed at shortening the load paths and for design02 , at simplifying the sections.

\section{Complexity for aero-engine structures}

The approach developed in Section 3 was used to quantify complexity for aero-engine structures. Two compressor structures, designed within EU research projects VITAL (European Commision Transport Research and Innovation Monitoring and Information System 2005) and LEMCOTEC (European Commision Community Research and Development Information Service 2011), were chosen for evaluation. Both structures are part of compressor modules, satisfying the same general set of functions despite belonging to two different engines. Therefore, the structures belong to the same class of products, commonly termed as cold structures (cold since the location
Fig. 8 Mechanical connectivity of sections for design-01 and design-02. Rows and columns of the matrices include all sections from both the designs. Orange cells indicate connections between sections in the respective rows and columns. Gray cells are connections for the same sections which are not considered

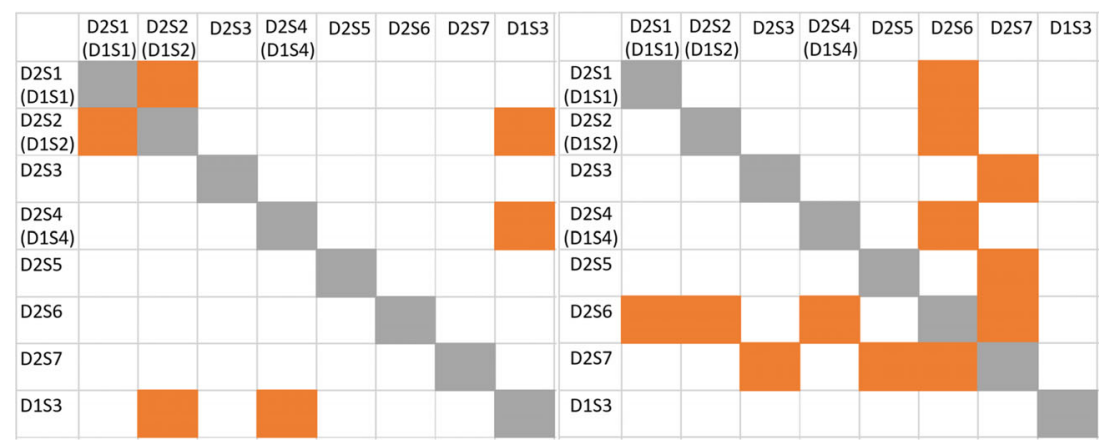


Table 3 Total complexity for design-01 and design-02

\begin{tabular}{ll}
\hline Design & Overall complexity C \\
\hline Design-01 & $C=C 1+C 2 C 3=39.6+2 \times 0.56=40.72$ \\
Design-02 & $C=C 1+C 2 C 3=80.3+4 \times 0.83=83.62$ \\
\hline
\end{tabular}

is before the burner, which is a relatively colder region). A complexity metric will enable comparing the structures both in terms of their geometry and their main function of load transfer. The VITAL engine structure is designated as structure-01 while the LEMCOTEC structure is designated as structure-02.

\subsection{1 complexity}

The different sections of structure-01 and structure-02 are marked in Figs. 9 and 10 respectively. Structure01 has 31 sections while structure- 02,33 . Together, the structures have 41 sections. Figure 11 shows the simplified representations for the sections. In the figure, 6 unique sectional representations, the corresponding PAG and the $C 1$ complexities are given. Simplifications have been performed such that the sectional geometry comprises only the relevant characteristics. For example, consider the vane, depicted as a plate with five dimensions in the upper left hand corner. The geometry is sufficient to conduct load transfer even though the actual aerodynamic geometry is more detailed as can be observed from Figs. 9 and 10. Simplifications performed similarly to the vane have been done for all other sections. The total $C 1$ complexity for both the engine structures are also given in Fig. 11.

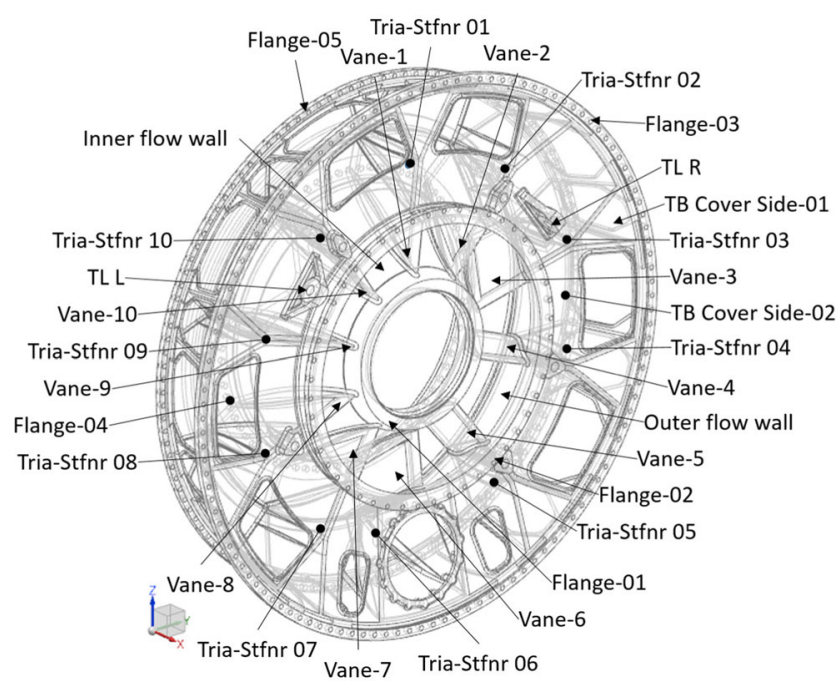

Fig. 9 Structure-01 with its sections. Marking lines that end with dots indicate sections hidden from view and lines that end with arrows indicate sections immediately visible
Even though the two structures have comparable number of sections, their component complexities differ significantly. Structure-02 has a higher $C 1$ than structure-01 due to the additional sections (outer stiffeners) present. The different $C 1$ complexities for structures in the same class is also indicative of their parent engine architectures. Thus, $C 1$ complexity can be used to classify and group structures based on their geometries and engine architectures.

\subsection{C2 complexity}

For calculating the interface complexity $C 2$, three typical loading scenarios for each compressor structure are considered. Loading details and detailed loading arrangements for all scenarios are given in Fig. 12. All scenarios have the same boundary conditions; however, the flanges where loads are applied and the type of loading at the flanges change under each scenario.

Following the procedure detailed in Section 3.2, the resulting load path sectional adjacency matrices are shown in Fig. 13. From the matrices, the load path for a given loading scenario can be traced. For example, during LS1 for strucutre-01, the applied load on flange-04 is transferred to flange-05 through the inner flow wall, vanes, triangular sitffeners, and torsion box cover sections (in Fig. 13, the row-column transitions "Flange-04 - Inner flow wall," "Inner flow wall - Vane 1-10," "Vane 1-10 - Tria-Stfnr 0110," "Tria-Stfnr 01-10 - TB Cover Side-02," and "TB Cover Side-02 - Flange-05"). It can be observed that sectional connections involving vanes ("Vane 1-10 -Tria-Stfnr 0110 " in structure-01 and "Vane 1-8 - Outer-Stiffener 1-8" in structure-02) are active in all loading scenarios. In both the structures, the connection between the loading and support flanges is achieved by means of the vanes. Therefore, reducing the number of vanes can reduce both $C 1$ and $C 2$ complexity of the structure.

Table 4 lists the computed $C 2$ complexities based on the load path sectional adjacency of Fig. 13. For structure02 , the interface complexity during each loading scenario is nearly the same. Structure-02 design responds to different loading scenarios effectively, involving the same number of section-section transitions during each loading scenario. Under LS1 and LS2, the $C 2$ complexities for structure01 are equal. This indicates the similarity of load paths during the concerned scenarios. The same phenomena can be observed for structure-02 during LS1 and LS2.

\subsection{C3 complexity}

The sectional mechanical connectivity matrices for structure-01 and structure-02 are shown in Fig. 14. The computed topological complexity $C 3$ is given in Table 5 . 

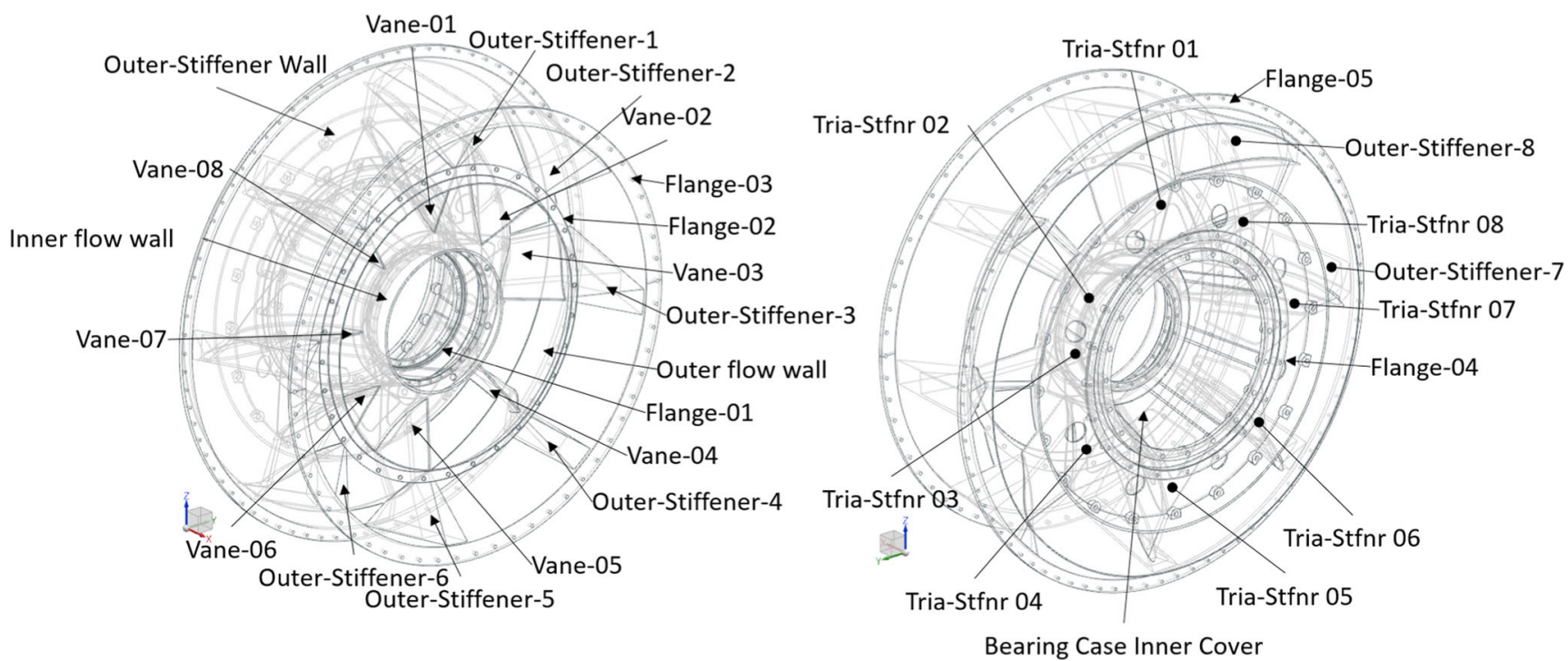

Fig. 10 Structure-02 with its sections. Views from both front and rear directions are shown for easier understanding of sections. Marking lines that end with dots indicate sections hidden from view and lines that end with arrows indicate sections immediately visible

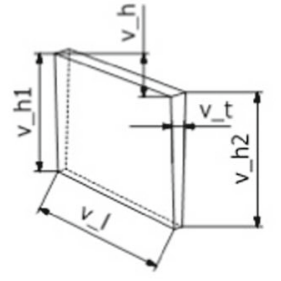

Vanes

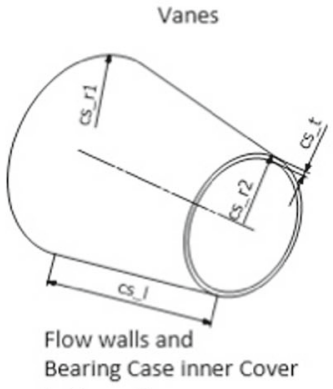

In Figure 9

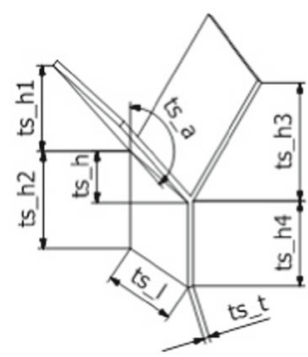

Triangular stiffeners (marked as Tria-Stfnr in Figure 8 and Figure 9)

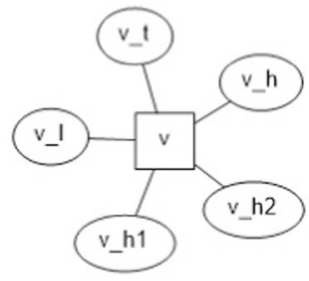

$\mathrm{C} 1=12.08$

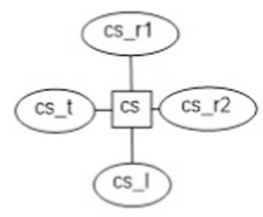

$C 1=9.89$

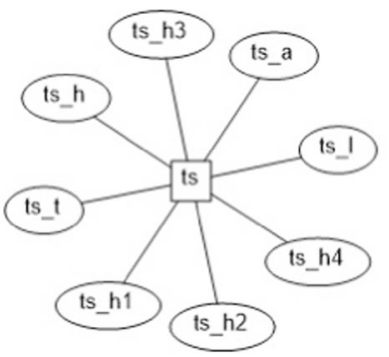

$C 1=18.68$

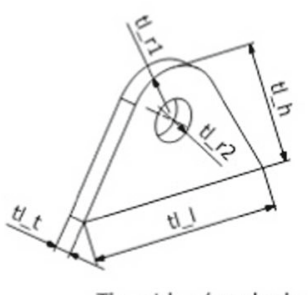

Thrust lug (marked as

TL R and TL L in Figure 8)

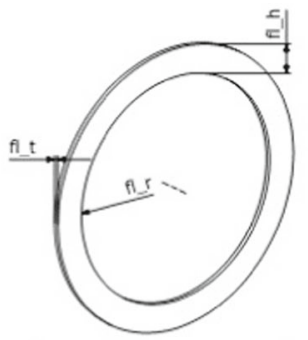

Flanges and TB Cover sides 01 and 02 in Figure 8

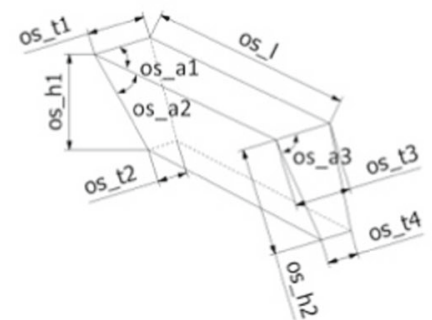

Outer stiffeners

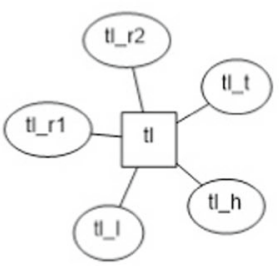

C1 $=12.08$

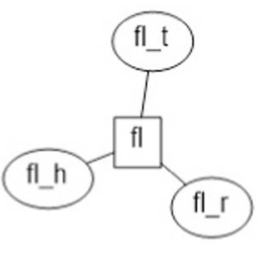

$\mathrm{C} 1=7.69$
Structure-01,

total $\mathrm{C} 1=\mathbf{4 0 5 . 3 9}$

Structure-02,

total $\mathrm{C} 1=508.66$

Fig. 11 PAG and $C 1$ for the different sections of structure- 01 and structure-02 


\section{Load Details for Structure-01 Loading Scenarios (LS)}

FX load on Flange-01

Load Details for Structure-02 Loading Scenarios (LS)

FX load on flange-01

FX load on flange-04

$\mathrm{MX}, \mathrm{MY}$ and $\mathrm{MZ}$ loads on flange-02

\section{Structure-01 Loading Arrangements}

Outer flanges (nos. 03 and 05) are attached to fixed dummies in all LS

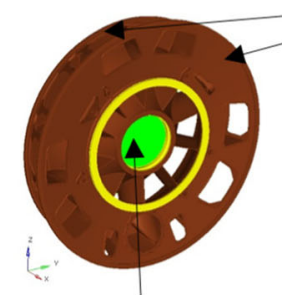

Constraint equations (CEs) for applying LS1 loads on flange- 04

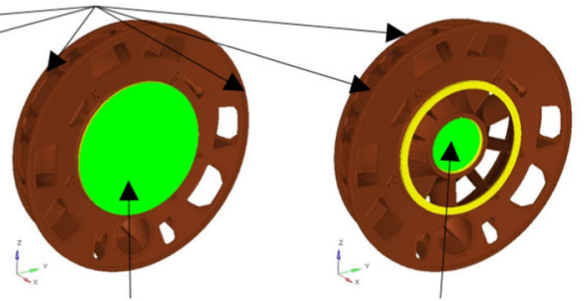

Constraint equations (CEs) for applying LS2 loads on flange- 02
Constraint equations (CEs) for applying LS3 loads on flange-01

\section{Structure-02 Loading Arrangements}

Outer flanges (nos. 03 and 05) are attached to fixed dummies in all LS

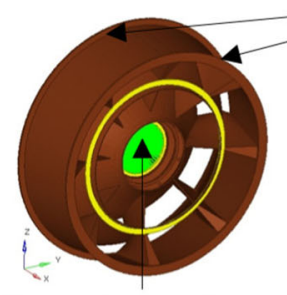

Constraint equations (CEs) for applying LS1 loads on flange-01

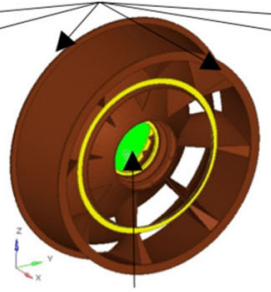

Constraint equations (CEs) for applying LS2 loads on flange-04

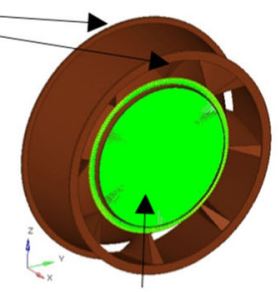

Constraint equations (CEs) for applying LS3 loads on flange-02

Fig. 12 Loading scenarios for structure-01 and structure-02. Regions marked in yellow do no participate in optimization

Load path sectional adjacency for structure-01

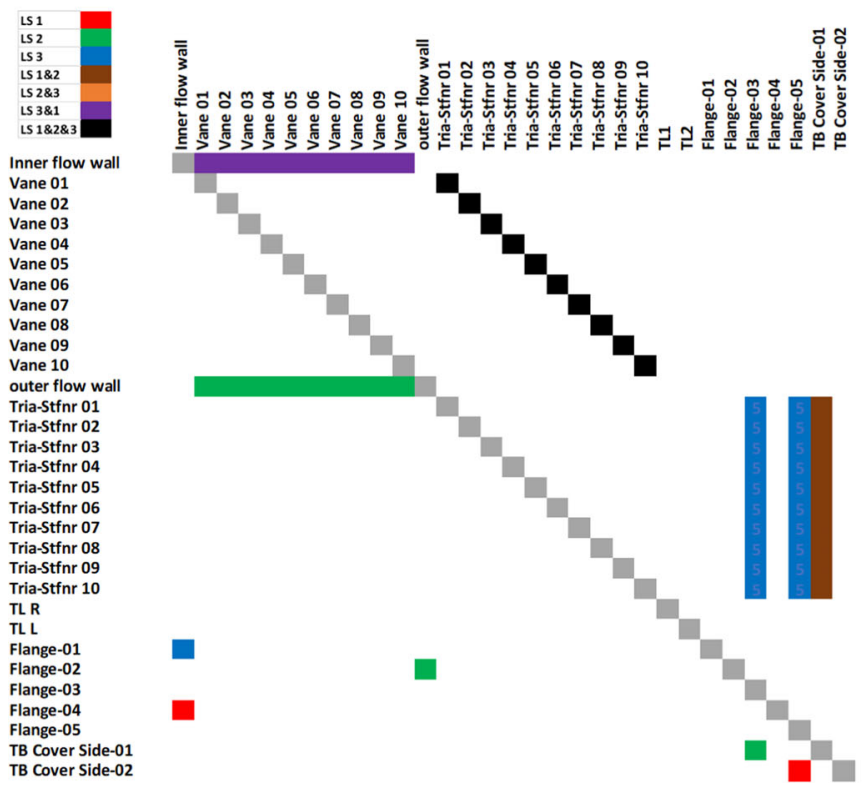

Fig. 13 Sectional adjacency under the considered loading scenarios for structure-01 and structure-02. Color coding indicates sectional participation in load transfer during the loading scenarios. For example, black cells indicate that the sections in the corresponding rows and columns participate in the load transfer under all loading scenarios. For
Load path sectional adjacency for structure-02

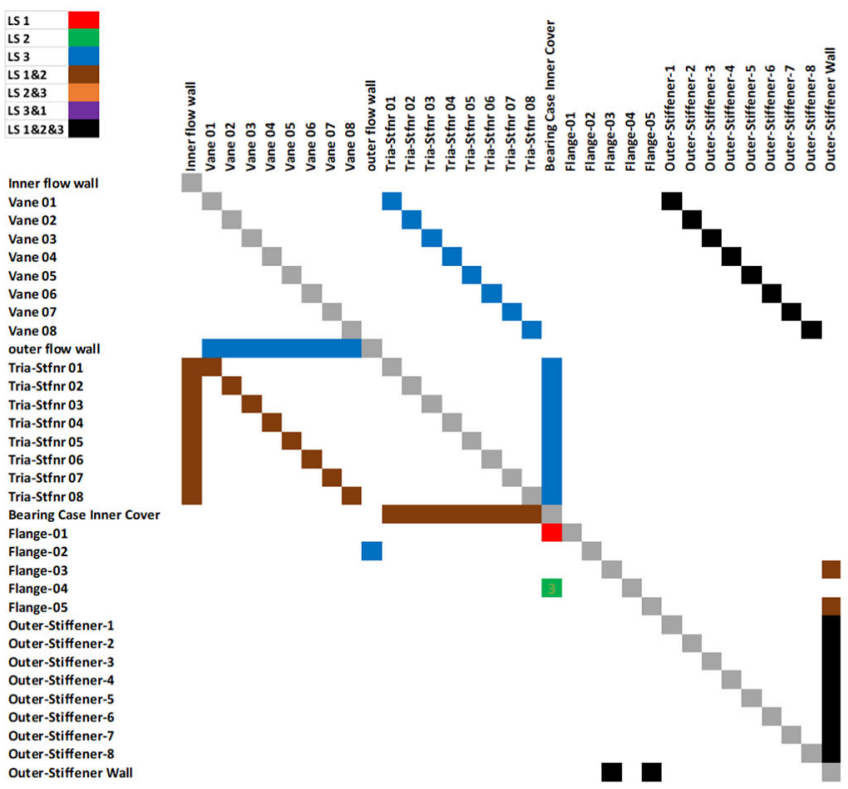

structure-01, this means "Vane 01-Tria-Stiffnr 01" transition is always utilized in all loading scenarios and for structure-02, "Vane 01-OuterStiffener-1" connection is utilized in all loading scenarios. Gray cells are connections for the same sections which are not considered 
Table $4 C 2$ Complexities for the considered loading scenarios for structure-01 and structure- 02

\begin{tabular}{lllll}
\hline Design & $C 2$ in LS1 & $C 2$ in LS2 & $C 2$ in LS3 & Total $C 2$ \\
\hline Structure-01 & 16.00 & 16.00 & 20.50 & 52.50 \\
Structure-02 & 22.00 & 22.00 & 21.50 & 65.50
\end{tabular}

The topological complexities for both structures are nearly equal implying that the sectional layouts in the structures are very similar. Comparable values of $C 3$ also indicate the architectural similarity of the structures' parent components. The structures are part of axial flow compressor modules that operate by transferring fluid through an annulus (the space formed between two concentric cylinders). The structures should also accommodate an annulus which is constructed by the circumferential arrangement of a number of vanes between an inner and an outer flow wall. Without a change in the architecture of the parent component (for example, to a centrifugal compressor), topological complexity $C 3$ for the structures may not change significantly during design improvements.

\subsection{Total complexity for the engine structures}

The total complexities for structure-01 and structure-02 are given in Table 6 . The proportion of components complexity $C 1$, and interface and topological complexities $C 2 C 3$, is nearly the same in both structures; $87 \%$ and $13 \%$ respectively. This is due to the comparable number of sections and layout of the sections in the structures.
Table 5 Topological complexity $C 3$ for structure- 01 and structure- 02

Design

Structure-01

1.14

Structure-02

Nearly, equal share of constituent complexities is indicative of the structures' architectural similarity and supports the grouping of the structures into the same class.

Despite the similarities in functions and the number of sections, the total complexity differs for both engine structures. Structure- 02 is $25 \%$ more complex than structure- 01 . A number of researchers have used complexity to estimate product development effort (Bashir and Thomson 2001; Sinha et al. 2013; Zhang and Thomson 2018). Complexity is correlated with development effort in man-hours for a number of products and projects, and a power law is recommended for the correlation. The relationship between complexity and product development effort takes the following general form:

$E \propto C^{b}$

where $E$ is the development effort in man-hours, $b$ is a constant, and $C$ is the total complexity. Based on studies conducted at a hydroelectric generator company, Zhang and Thomson (2018) estimate $b$ as 1.786. Sinha et al. (2013) estimate $b$ as 1.69 based on the time it took for human subjects to assemble molecular study kits of different total complexities. Adopting $b$ as 1.75 , a $25 \%$ difference in complexity leads to $48 \%$ difference in development

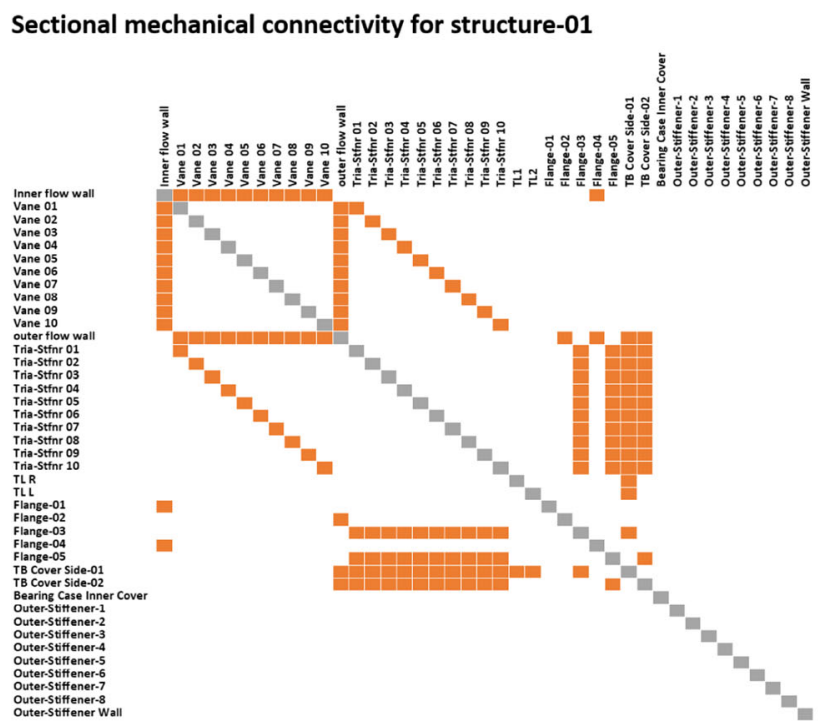

Sectional mechanical connectivity for structure-02

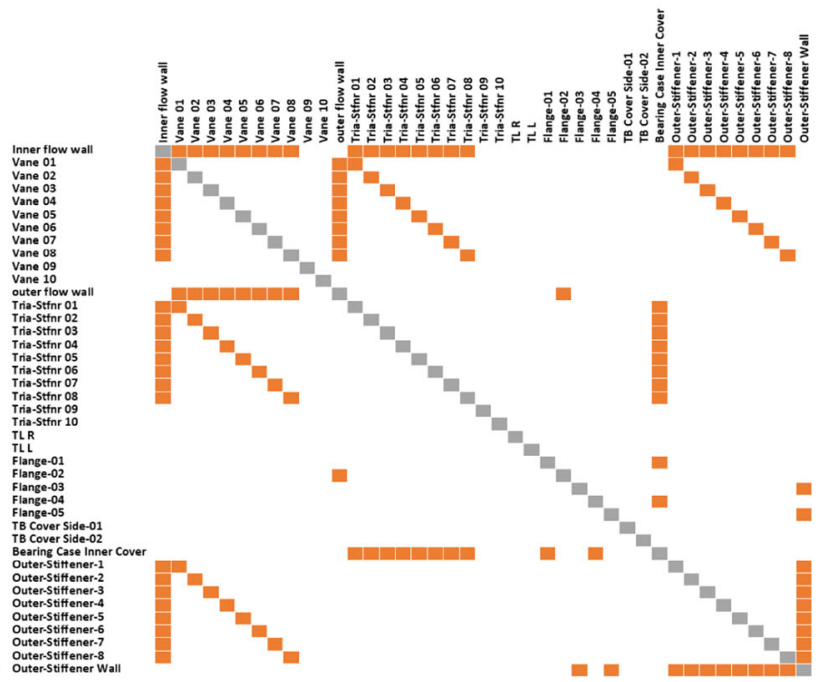

Fig. 14 Mechanical connectivity of sections for structure-01 and structure-02. Orange cells indicate connections between sections in the respective rows and columns. Gray cells are connections for the same sections which are not considered 
Table 6 Total complexity for structure- 01 and structure- 02

\begin{tabular}{ll}
\hline Design & Overall complexity C \\
\hline Structure-01 & $C=C 1+C 2 C 3=405.39+52.5 \times 1.14=465.24$ \\
Structure-02 & $C=C 1+C 2 C 3=508.66+65.5 \times 1.13=582.66$ \\
\hline
\end{tabular}

costs. Such calculations can be helpful in obtaining cost projections for future product development efforts.

\section{Conclusion}

The development of integrated architecture structures relies significantly on individual experience. Estimates of complexity (affecting development time) is subjective and can be erroneous. A systematic and quantitative metric for complexity can assist the design and optimization processes tremendously.

In this work, we propose a complexity metric for integrated architecture structures that can be used to compare design alternatives. The developed metric is founded on existing metrics developed for assessing complexity at the system and component levels. It is composed of three constituents (a component complexity $C 1$, an interface complexity $C 2$, and a topological complexity $C 3$ ) that together provide a measure of total complexity. To enable computations for integrated architecture structures, the latter are partitioned into different sections (regions). Interactions among sections are estimated as load paths, identified by means of topology optimization simulations. Two aero-engine static structures satisfying similar functions but were used to demonstrate complexity evaluations in order to illustrate how the latter can be considered in the design and optimization processes.

Integrated architecture structures are often partitioned into segments and weld-fabricated. The partitioning and manufacturing method of the segments should be taken into account during early design stages. Design is also affected by the gas flow path aspect of the structure's functions. Future work could focus on a unified design structure complexity metric that takes into account both manufacturing and functional aspects of the design.

\section{Replication of results}

No results are presented.

Funding information This work has financially been supported by NFFP, the national aeronautical research programme, jointly funded by the Swedish Armed Forces, Swedish Defense Materiel Administration (FMV) and Swedish Governmental Agency for Innovation Systems (VINNOVA).

\section{Compliance with ethical standards}

Conflict of interest The authors declare that they have no conflict of interest.

Open Access This article is distributed under the terms of the Creative Commons Attribution 4.0 International License (http:// creativecommons.org/licenses/by/4.0/), which permits unrestricted use, distribution, and reproduction in any medium, provided you give appropriate credit to the original author(s) and the source, provide a link to the Creative Commons license, and indicate if changes were made.

\section{References}

Ameri F, Summers JD, Mocko GM, Porter M (2008) Engineering design complexity: an investigation of methods and measures. Res Eng Des 19(2-3):161-179. https://doi.org/10.1007/s00163-0080053-2

Bashir HA, Thomson V (2001) Models for estimating design effort and time. Des Stud 22(2):141-155. https://doi.org/10.1016/S0142694X(00)00014-4

Chu D, Strand R, Fjelland R (2003) Theories of complexity. Complexity 8(3):19-30. https://doi.org/10.1002/cplx.10059

ElMaraghy W, ElMaraghy H, Tomiyama T, Monostori L (2012) Complexity in engineering design and manufacturing. CIRP Ann 61(2):793-814. https://doi.org/10.1016/j.cirp.2012.05.001

European Commision Community Research and Development Information Service (2011) Low emissions core-engine technologies (LEMCOTEC). https://cordis.europa.eu/project/rcn/100239_ en.html, Accessed 12 Sept 2018

European Commision Transport Research and Innovation Monitoring and Information System (2005) VITAL environmentally friendly aero-engine. https://trimis.ec.europa.eu/project/ environmentally-friendly-aero-engine\#tab-outline, Accessed 12 Sept 2018

Hazelrigg GA (1998) A framework for decision-based engineering design. J Mech Des 120(4):653-658

Hölttä-Otto K, de Weck O (2007) Degree of modularity in engineering systems and products with technical and business constraints. Concurr Eng 15(2):113-126. https://doi.org/10.1177/ $1063293 \times 07078931$

Jiao J, Simpson TW, Siddique Z (2007) Product family design and platform-based product development: a state-of-the-art review. J Intell Manuf 18(1):5-29. https://doi.org/10.1007/s10845-0070003-2

Kelly DW, Reidsema CA, Lee MCW (2011) An algorithm for defining load paths and a load bearing topology in finite element analysis. Eng Comput 28(2):196-214. https://doi.org/10.1108/ 02644401111109231

Kim G, Kwon Y, Suh ES, Ahn J (2016) Analysis of architectural complexity for product family and platform. J Mech Des 138(7):071401-071401. https://doi.org/10.1115/1.4033504

Lindemann U, Maurer M, Braun T (2009) Structural complexity management: an approach for the field of product design, vol c2009. Springer, Berlin

Maurer M, Schneller R, Omer M (2014) A survey on complexity management in systems engineering. In: 8th Annual IEEE international systems conference. SysCon 2014 - proceedings, pp $180-187$

Mikkola JH (2006) Capturing the degree of modularity embedded in product architectures. J Prod Innov Manag 23(2):128-146. https://doi.org/10.1111/j.1540-5885.2006.00188.x 
Min G, Suh ES, Hölttä-Otto K (2015) System architecture, level of decomposition, and structural complexity: analysis and observations. J Mech Des 138(2):021102-021102. https://doi.org/10. $1115 / 1.4032091$

Naito T, Kobayashi H, Urushiyama Y, Takahashi K (2011) Introduction of new concept $\mathrm{u}^{*}$ sum for evaluation of weight-efficient structure. SAE Int J Passenger Cars - Electron Electr Syst 4(1):30-41. https://doi.org/10.4271/2011-01-0061

Pahl G, Wallace K, Blessing L, SpringerLink (2007) Engineering design: a systematic approach, 3rd edn. Springer, London

Pugh S (1990) Total design: integrated methods for successful product engineering. Addison-Wesley

Raja V (2016) On integrated product architectures: representation, modelling and evaluation. Licentiate Thesis Chalmers University of Technology, Göteborg, Sweden

Raja V, Isaksson O (2015) Generic functional decomposition of an integrated jet engine mechanical sub system using a configurable component approach, vol 2. IOS Press, Delft, pp 337-346. Advances in transdisciplinary engineering: transdisciplinary lifecycle analysis of systems

Shah JJ, Runger G (2013) What is in a name? On the misuse of information theoretic dispersion measures as design complexity metrics. J Eng Des 24(9):662-680. https://doi.org/10.1080/09544828. 2013.824952

Shinobu M, Okamoto D, Ito S, Kawakami H, Takahashi K (1995) Transferred load and its course in passenger car bodies. JSAE Rev 16(2):145-150. https://doi.org/10.1016/0389-4304(95)00010-5
Sinha K, de Weck OL (2016) Empirical validation of structural complexity metric and complexity management for engineering systems. Syst Eng 19(3):193-206. https://doi.org/10.1002/sys.21356

Sinha K, Omer H, De Weck OL (2013) Structural complexity: quantification, validation and its systemic implications for engineered complex systems. In: Proceedings of the international conference on engineering design. ICED 4 DS75-04, pp 189198

Sobek I, Durward K, Ward AC, Liker JK (1999) Toyota's principles of set-based concurrent engineering. Sloan Manage Rev 40(2):67

Suh NP (2005) Complexity: theory and applications. Oxford University Press, New York

Summers JD, Shah JJ (2010) Mechanical engineering design complexity metrics: size, coupling, and solvability. J Mech Des 132(2):021004. https://doi.org/10.1115/1.4000759

Urbanic RJ, ElMaraghy W (2009) A design recovery framework for mechanical components. J Eng Des 20(2):195-215. https://doi.org/10.1080/09544820701802261

Weber C (2005) What is "complexity"? Melbourne, Australia. In: The 15th international conference on engineering design, pp 292-293

Zhang X, Thomson V (2018) A knowledge-based measure of product complexity. Comput Indus Eng 115:80-87. https://doi.org/10. 1016/j.cie.2017.11.005

Publisher's note Springer Nature remains neutral with regard to jurisdictional claims in published maps and institutional affiliations. 